\title{
FITOSSOCIOLOGIA E SUPRESSÃO DE PLANTAS DANINHAS SOB EFEITO DA SOLARIZAÇÃO E COBERTURA COM CAPIM-ELEFANTE
}

\author{
Ana Paula Werkhausen Witter ${ }^{1}$, Marcos André Nohatto², Bruno Leffa Borges ${ }^{1}$, Jéssica Fernandes \\ Kaseker $^{3}$, Eliete de Fátima Ferreira da Rosa ${ }^{4}$, Leonardo Geremias Madeira ${ }^{1}$, Alessandro Porto
} Fermiano $^{1}$

\begin{abstract}
RESUMO - A técnica de solarização e da cobertura vegetal são estratégias de manejo de plantas daninhas importantes para reduzir a mão-de-obra e outras limitações impostas pelo controle mecânico, porém a eficiência e resposta na composição florística desses métodos varia conforme manejo realizado na agricultura familiar. Dessa forma, o objetivo com esse trabalho é avaliar o efeito supressivo da solarização e cobertura com capimelefante (Pennisetum purpureum Schum.) sobre a emergência de plantas daninhas, bem como identificar e quantificar as espécies daninhas através do levantamento fitossociológico. O experimento foi conduzido a campo no ano agrícola 2017/2018, utilizando delineamento experimental em blocos casualizados e quatro repetições. Os tratamentos foram dispostos em esquema fatorial, onde o fator A compara a solarização (presença e ausência); e o fator B a cobertura com capim-elefante (sem e com). Realizou-se o levantamento fitossociológico e avaliação da massa seca da parte aérea das plantas daninhas em resposta aos tratamentos. A adição do capim-elefante na superfície do solo atua como supressor da diversidade de plantas daninhas, como Ageratum conyzoides, Urochloa plantaginea, Galinsoga parviflora, Polygonum persicaria, Spermacoce latifolia, Sida sp. e Stachys arvensis, enquanto que a solarização, aumenta o valor de importância do Cyperus sp. nas parcelas sem a utilização da cobertura vegetal. Ainda, a solarização realizada por 20 dias não foi eficiente para o controle das plantas daninhas.
\end{abstract}

Palavras chave: levantamento fitossociológico, mulching, Pennisetum purpureum.

\section{PHYTOSOCIOLOGY AND SUPPRESSION OF WEEDS UNDER THE EFFECT OF SOLARIZATION AND NAPIER GRASS COVER}

\begin{abstract}
The solarization techniques and vegetation cover are important weed management strategies to reduce manpower and other limitations imposed by mechanical control, but the efficiency and response in the floristic composition of these methods varies according to the management performed in family farming. Thus, the aim of this study is to evaluate the suppressive effect of soil solarization and the cover with napier grass (Pennisetum purpureum) on weeds emergence, as well as to identify and quantify the present weeds through the phyto-sociological survey. The experiment was conducted under field conditions in the agricultural year 2017/2018, using a completely randomized block design with four replications. The treatments were arranged in a factorial scheme, where factor A compares solarization (presence and absence); and factor $B$ the cover with napier grass (without and with). In response to the treatments, the phyto-sociological survey and the evaluation of the shoot dry mass of the weeds were conducted. The napier grass addition to the soil surface acts as suppressor of diversity weeds, such Ageratum conyzoides, Urochloa plantaginea, Galinsoga parviflora, Polygonum persicaria, Spermacoce latifolia, Sida spp. and Stachys arvensis. The solarization increases the importance value of Cyperus sp. in the plots without the use of vegetation cover. Also, the solarization performed for 20 days was not efficient for weed control.
\end{abstract}

Keywords: phyto-sociological survey, mulching, Pennisetum purpureum.

\footnotetext{
${ }^{1}$ Discente do curso de Engenharia Agronômica. IFC Campus Santa Rosa do Sul. ana_werkhausen@outlook.com; bruno.borges99@hotmail.com; leo.geremiasmadeira@hotmail.com; alessandroporto147@gmail.com.

${ }^{2}$ Eng. Agr. Dr. em Fitossanidade (Herbologia). IFC Campus Santa Rosa do Sul. marcos.nohatto@ifc.edu.br (autor para correspondência). ${ }^{3}$ Enga.Agr. Dra. em Manejo do Solo. Universidade do Estado de Santa Catarina. IFC Campus Santa Rosa do Sul. jessica.kaseker@ifc.edu.br.

${ }^{4}$ Enga. Agr. Dra. em Ciência do Solo. Universidade do Estado de Santa Catarina. IFC Campus Santa Rosa do Sul. eliete.rosa@ifc.edu.br.
} 


\section{INTRODUÇÃO}

No cultivo agroecológico é proibido a utilização de herbicidas químicos sintéticos, em função do impacto toxicológico e ambiental desses produtos. Dessa maneira, o controle de plantas daninhas nesse sistema de produção, baseia-se, na maioria das vezes, no manejo mecânico com uso de enxadas e cultivadores. Essa estratégia possui uma série de limitações como baixo rendimento, maior dificuldade de eliminação das daninhas na linha de plantio, eventuais danos causados às raízes superficiais da cultura, a dispersão de propágulos para áreas não infestadas, exposição do solo a erosão e formação de camadas adensadas no solo (Oliveira Jr. et al., 2011).

As desvantagens associadas ao controle mecânico de plantas daninhas dificultam o manejo das culturas e limitam a expansão do cultivo agroecológico. Esse fato incentiva a comunidade científica a avaliar outras estratégias para o controle de plantas daninhas, que mantenham baixo impacto ambiental e também sejam compatíveis com a realidade do produtor.

Nesse contexto, a utilização de cobertura vegetal tem sido relatada como alternativa, reduzindo a germinação e estabelecimento das espécies (Trezzi \& Vidal, 2004). Estudo conduzido por Correia e Durigan (2004), constatou que a cobertura do solo com 5, 10 e $15 t_{\text {ha }}^{-1}$ de palha de cana inibiu a emergência de plântulas das espécies Brachiaria decumbens e Sida spinosa. No entanto, a presença da cobertura incrementou a emergência de plântulas de Ipomoea quamoclit, indicando que a resposta foi dependente da espécie presente no banco de sementes.

Outra estratégia de manejo das plantas daninhas refere-se a solarização, que consiste na colocação de um filme plástico transparente sobre solo umedecido, durante o período do ano de maior radiação solar (Patrício et al., 2007). Essa técnica de desinfestação também tem sido utilizada para o controle de nematoides como Meloidogyne javanica (Klein et al., 2012) e fitopatógenos como Fusarium oxysporum (Wong et al., 2011), Verticillium dahliae (Kanaan et al., 2015), Sclerotinia sclerotiorum e S. rolfsi (Rocha \& Carneiro, 2016).

Tanto a solarização como a cobertura vegetal tem grande potencial de uso para agricultura agroecológica, porém fatores como tipo de plástico e/ou cobertura, época do ano, duração do mulching podem influenciar na eficiência dessas estratégias de manejo de plantas daninhas, além do efeito sobre a composição florística (Candido et al., 2012; Ferreira et al., 2013; Talebi \& Golparvar, 2013).

Diante disso, o objetivo com esse trabalho é avaliar o efeito supressivo da solarização e cobertura com capim-elefante sobre a emergência de plantas daninhas, bem como identificar e quantificar as espécies daninhas presentes através do levantamento fitossociológico.

\section{MATERIALE MÉTODOS}

O experimento foi conduzido em área experimental pertencente ao Instituto Federal Catarinense - Campus Santa Rosa do Sul durante o período de dezembro de 2017 a março de 2018. O solo do local foi classificado como Gleissolo Melânico (Embrapa, 1999) e apresenta as seguintes características químicas: $\mathrm{pH}$ em água, 6,5; \% M.O, 2,6; P, $52 \mathrm{mg} / \mathrm{dm}^{3} ; \mathrm{K}, 0,9 \mathrm{cmol} / \mathrm{dm}^{3}$; Ca trocável, 4,4 $\mathrm{cmol}_{\mathrm{c}} / \mathrm{dm}^{3} ; \mathrm{Mg}$ trocável, $1,7 \mathrm{cmol}_{\mathrm{c}} / \mathrm{dm}^{3}$; $\mathrm{H}+\mathrm{Al}, 2,7 \mathrm{cmol}_{\mathrm{c}} / \mathrm{dm}^{3}$; CTC, 9,7; Al trocável, $0 \mathrm{cmol}_{\mathrm{c}} /$ $\mathrm{dm}^{3}$. O bioma da região de estudo é caracterizado como Mata Atlântica. O clima da região é do tipo Cfa (Clima Subtropical Úmido) com temperatura média de $19^{\circ} \mathrm{C}$ e chuvas bem distribuídas ao longo do ano. A Figura 1 indica a precipitação total e temperatura média e mensais durante o período de condução do experimento.

Anteriormente a implantação do experimento o solo havia sido cultivado com aveia branca (Avena sativa). O delineamento experimental adotado foi blocos

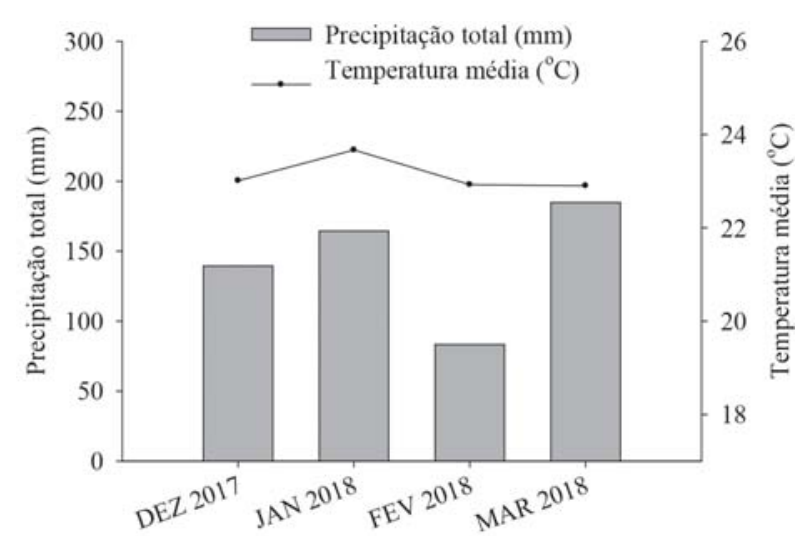

Figura 1 - Valores de precipitação total e temperatura média mensais registrados durante o período de condução do experimento (dezembro/2017 a março/2018) no IFC Campus Santa Rosa do Sul. 
casualizados, com quatro repetições, tendo cada unidade experimental área de $3,75 \mathrm{~m}^{2}$ (2,5m x 1,5m). Os tratamentos foram arranjados em esquema fatorial (2x2), onde o fator A comparou a solarização (presença e ausência); e o fator B a cobertura com capim-elefante (Pennisetum purpureum) (sem e com) $\left(4,8 \mathrm{~kg} / \mathrm{m}^{2}\right.$ da planta triturada fresca, formando camada de aproximadamente $5 \mathrm{~cm}$ ).

O filme plástico, transparente de 150 micras, foi inserido nas unidades experimentais determinadas com intuito de provocar a “solarização”. Inicialmente os canteiros foram preparados e nivelados e com o mínimo de torrões possíveis a fim de evitar bolsões de ar. Posteriormente, os canteiros foram irrigados e em seguida o plástico transparente foi colocado sobre a superfície do canteiro. Para garantir a permanência do plástico sobre o canteiro, esse foi prendido nas bordas com solo, sem prejuízo da área útil do canteiro. O tempo de solarização foi de 20 dias, realizado no mês de janeiro.

Transcorrido o período acima indicado, o plástico foi devidamente removido. Imediatamente após, foi realizado o monitoramento da temperatura da superfície do solo em triplicata por parcela, sendo empregado termômetro digital (tipo Espeto; Incoterm ${ }^{\circledR}$ ) e medição a $1 \mathrm{~cm}$ de profundidade (Figura 2). Na sequência houve a implantação da cobertura com capim-elefante nas unidades experimentais designadas.

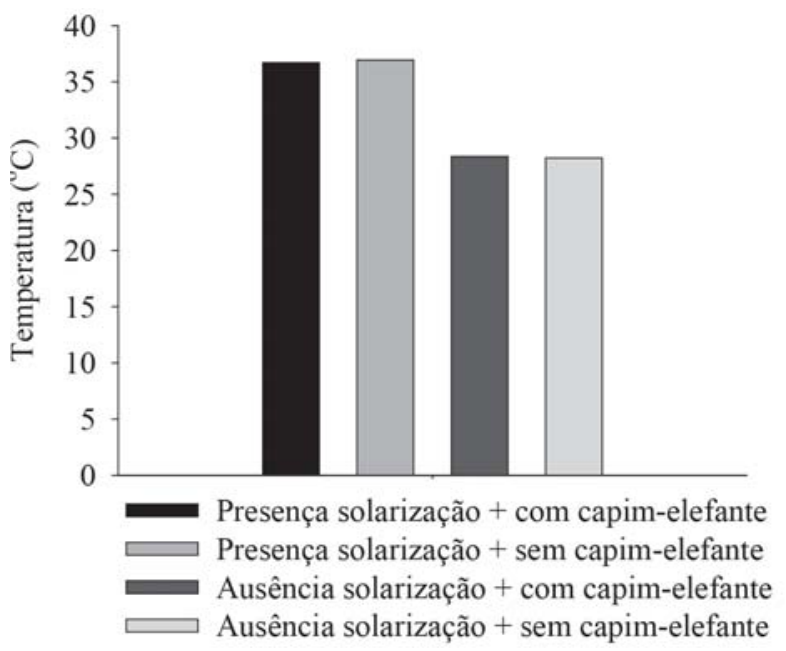

Figura 2 - Temperatura do solo, avaliada na profundidade de $1 \mathrm{~cm}$, realizada ao final da solarização, ou seja, 20 dias após a colocação do filme plástico transparente (150 micras).
Aos 10 e 40 dias após o final da solarização (DAS), ou seja, da retirada do plástico e implantação da cobertura vegetal, foram efetuadas amostragens das plantas daninhas nas parcelas. Para isso, arremessou-se, aleatoriamente, um quadro vazado de madeira com 0,4 m de lado e área interna de $0,16 \mathrm{~m}^{2}$, sendo uma repetição por parcela. Posteriormente, as plantas daninhas emergidas foram contabilizadas e identificadas. Esse tipo de avaliação permite determinar a composição florística, a estrutura de funcionamento, a dinâmica e a distribuição de uma determinada vegetação, em determinada área (Lamego et al., 2015).

A partir das informações coletadas, foram calculados os parâmetros fitossociológicos: frequência (índice da ocorrência das espécies em cada quadrado), densidade (índice da quantidade de indivíduos de uma mesma espécie em cada quadrado), abundância (concentração das espécies nos diferentes pontos da área total), frequência relativa, densidade relativa e abundância relativa (relaciona uma espécie a todas as demais encontradas nas áreas) e o índice de valor de importância, de acordo com Mueller-Dombois \& Ellenberg (1974), a partir das seguintes equações:

Frequência $=n^{\circ}$ de lançamento no qual foi detectada uma espécie / $\mathrm{n}^{\circ}$ total de lançamentos (1)

Frequência relativa $=($ Frequência de uma espécie x100) / (Frequência total) (2)

Densidade $=\mathrm{n}^{\circ}$ de indivíduos de uma espécie / área total amostrada (3)

Densidade relativa $=($ densidade de uma espécie $\mathrm{x} 100)$ / densidade total (4)

Abundância $=\mathrm{n}^{\circ}$ de indivíduos de uma espécie $/ \mathrm{n}^{\circ}$ de lançamentos no qual foi detectado uma espécie (5)

Abundância relativa = (abundância de uma espécie x 100) / Abundância total (6)

Índice de valor de importância (IVI) = frequência relativa + densidade relativa + abundância relativa (7)

Após a contagem e identificação para o levantamento fitossociológico foi realizado o corte das plantas daninhas rente ao solo para determinação da massa seca (MS - gramas) na área interna amostrada pelo quadrado vazado nas parcelas $\left(0,16 \mathrm{~m}^{2}\right)$. Para isso, colocou-se o material vegetal em estufa de circulação de ar quente a $60^{\circ} \mathrm{C}$ por período de \pm 72 horas. Esses dados foram submetidos à análise de variância $(\mathrm{p}<0,05)$. No caso de ser constatada significância estatística, foi feita 
comparação de médias entre tipos de cobertura pelo teste Tukey $(\mathrm{p}<0,05)$.

\section{RESULTADOS E DISCUSSÃO}

No primeiro levantamento fitossociológico (10 DAS) verificou-se a presença de 9 espécies distribuídas em 7 famílias, enquanto que na segunda análise da composição invasora, realizada aos 40 DAS, observouse 13 espécies distribuídas em 9 famílias (Figuras 3 e 4), sendo constatada predominância de Poaceae, com 4 espécies (Tabela 1 ).

A espécie Stachys arvensis, presente no primeiro levantamento, foi a única planta não detectada na segunda época de avaliação. Supõe-se que esse comportamento possivelmente esteja relacionado com a menor habilidade competitiva da espécie comparada as demais, embora não tenha sido feita essa avaliação no experimento. Além disso, espécies de inverno, caso da hortelã-das-roças, germinam durante os períodos de temperatura mais amenas, ou seja, são menos tolerantes a solarização comparada a espécies de verão (Elmore, 1991), o que ajuda a explicar a ausência da espécie aos 40 DAS.

Ao analisar o índice de valor de importância (IVI), observou-se, em geral, que este foi superior para a espécie Cyperus sp., principalmente onde não houve cobertura com capim-elefante, independente da solarização (Figuras 3 e 4). Tais resultados demonstram a característica de dominância que a espécie apresenta frente às demais, pois além de produzir sementes, também pode-se propagar por rizomas, tubérculos e bulbos (Kissmann \& Groth, 1997).
Ainda para o Cyperus sp., ao considerar a diferença no comportamento da espécie nas parcelas solarizadas em comparação com as não-solarizadas, quando não temos a cobertura vegetal, verifica-se maior IVI do Cyperus sp. para solarização (Figuras 3 e 4). Plantas dessa espécie demonstram maior resistência à solarização do que outras plantas que se desenvolvem no mesmo ambiente, como demonstrado em diversos estudos que resultaram em controle parcial da população infestante, ou mesmo não apresentaram efeitos significativos (Ricci et al., 2000; Candido et al., 2011).

Com relação a variável massa seca (MS), não houve interação entre a solarização e cobertura vegetal, somente o efeito simples desses fatores (Tabela 2). Aos 10 DAS, observou-se que as parcelas submetidas a solarização apresentaram menor MS, enquanto que aos 40 DAS ocorreu o contrário. Tal resultado indica, que nas condições que foram realizadas o estudo, a solarização apresenta um controle residual de curto prazo, ou seja, na segunda época de avaliação observa-se que a solarização potencializou a produção da MS, possivelmente devido à quebra de dormência de plantas daninhas presentes na área.

Estudos diversos têm demonstrado a efetividade da solarização no controle de plantas daninhas (El-Keblawy \&Al-Hamadi, 2009; Candido et al., 2012; Talebi \& Golparvar, 2013), semelhante ao verificado para a primeira avaliação (10 DAS) (Tabela 2). No entanto, a maioria dos trabalhos tem utilizado acima de 20 dias de solarização, ou seja, o tempo de duração da cobertura com o plástico possivelmente foi abaixo do necessário para o controle. Ao avaliar o efeito

Tabela 1 - Família, nome científico e nome comum das espécies identificadas na área experimental

\begin{tabular}{lll}
\hline Família & Nome científico & Nome comum \\
\hline Convolvulaceae & Ipomoea sp. & Corda-de-viola \\
Poaceae & Digitaria sp. & Milhã \\
Cyperaceae & Cyperus sp. & Tiririca \\
Malvaceae & Sida sp. & Guanxuma \\
Amaranthaceae & Amaranthus sp. & Caruru \\
Asteraceae & Galinsoga parviflora Cav. & Picão-branco \\
Poaceae & Cynodon dactylon (L.) Pers. & Grama seda \\
Lamiaceae & Stachys arvensis L. & Hortelã-das-roças \\
Poaceae & Urochloa plantaginea (Link) R.D. Webster & Papuã \\
Poaceae & Echinochloa sp. & Capim-arroz \\
Asteraceae & Ageratum conyzoides L. & Picão-roxo \\
Commelinaceae & Commelina sp. & Trapoeraba \\
Rubiaceae & Spermacoce latifolia Aubl. & Erva-quente \\
Polygonaceae & Polygonum persicaria L. & Erva de bicho \\
\hline
\end{tabular}


da solarização durante dois meses, Setyowati et al. (2017) também não observou eficiência para o controle de plantas daninhas, com exceção do Amaranthus gracillis. Tais autores indicam que as plantas daninhas anuais, caso da espécie citada, são menos resistentes à solarização comparadas as plantas perenes, uma vez que essas possuem órgãos vegetativos presentes no solo com reserva capazes de germinar após o período de estresse.

O princípio da solarização coloca que o calor intenso e a umidade podem aumentar a germinação, mas também causar a morte das plantas daninhas emergentes que não toleram o excesso de calor, o que resulta em diminuição significativa da quantidade das invasoras presentes na área (Arora \& Yaduraju, 1998).

O menor tempo de solarização, além de não controlar as daninhas, acredita-se que favoreceu a quebra de dormência pela combinação da alta temperatura e amplitude térmica natural no período de condução do experimento, aumentando a densidade populacional do Cyperus sp., especialmente na ausência da cobertura vegetal (Figuras 3 e 4). Essa espécie é caracterizada por apresentar dormência de seus propágulos, sendo que temperaturas diurnas/noturnas de $38-32^{\circ} \mathrm{C}$ são ideais para germinação (Thullen \& Keeley, 1979), o que corrobora com os resultados obtidos, uma vez que tais temperaturas também foram detectadas nas parcelas solarizadas (Figura 1).

Marenco \& Lustosa (2000) demonstram também resultados semelhantes de indução da germinação de plantas daninhas com poucas semanas de solarização, porém com a espécie Commelina benghalensis. Além dela, há relato do estímulo da solarização para Melilotus sulcatus (Candido et al., 2011), bem como efeito nulo da técnica sobre Avena sterilis (Horowitz, 1983), indicando que a espécie-alvo, além da temperatura e tempo de duração da cobertura com o plástico são fatores importantes para serem considerados para avaliação da eficiência da solarização.

Em ambos os períodos de avaliação, observouse que a utilização do capim-elefante reduziu a MS (Tabela 2), que demonstra a importância da cobertura vegetal para supressão de plantas, além da redução
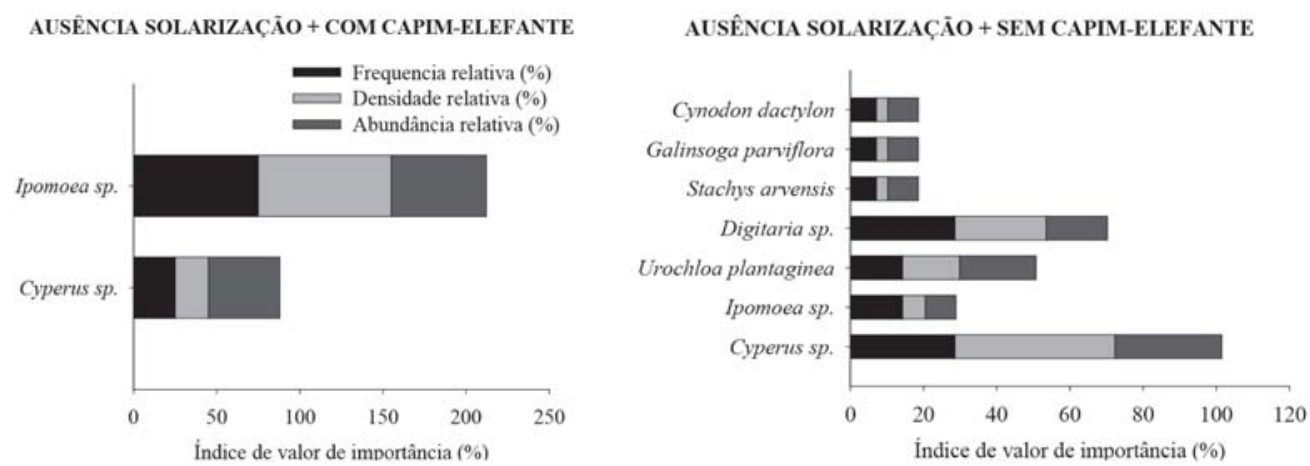

PRESENÇA SOLARIZAÇĀO + COM CAPIM-ELEFANTE

PRESENÇA SOLARIZAÇÃO + SEM CAPIM-ELEFANTE
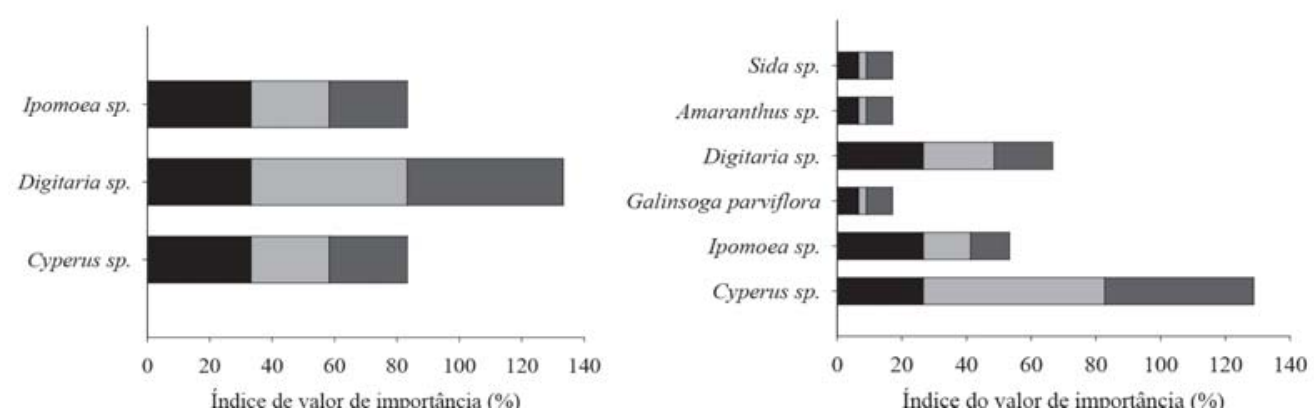

Figura 3 - Índice de valor de importância das plantas daninhas emergidas em resposta aos tratamentos experimentais (presença e ausência da solarização; com e sem capim-elefante) avaliado aos 10 dias após o final da solarização. 

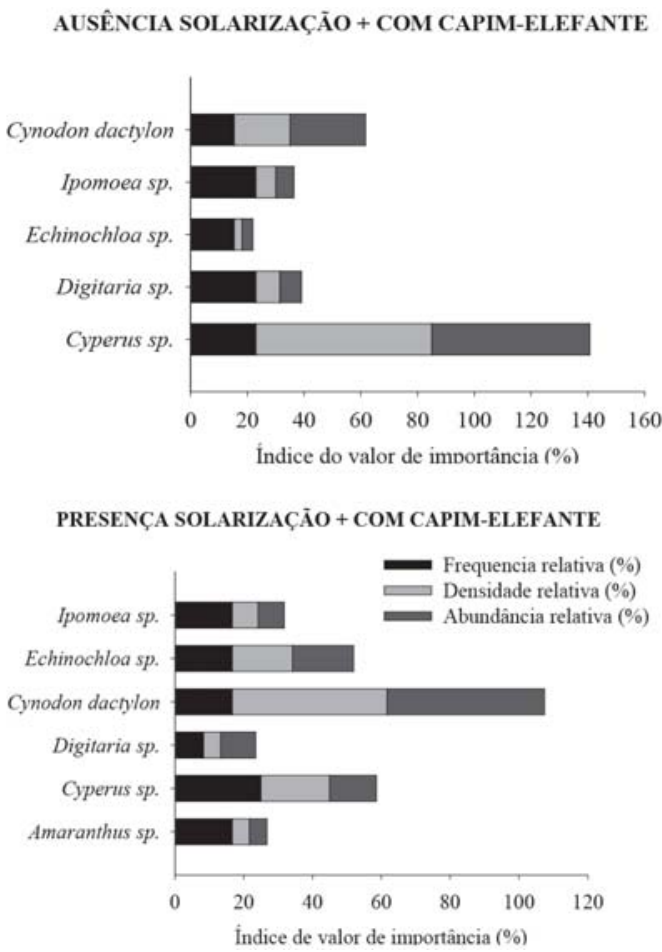

AUSÊNCIA SOLARIZAÇÃO + SEM CAPIM-ELEFANTE

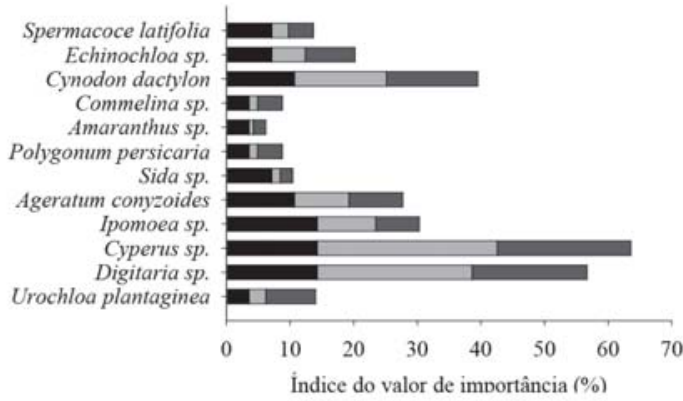

PRESENÇA SOLARIZAÇÃO + SEM CAPIM-ELEFANTE

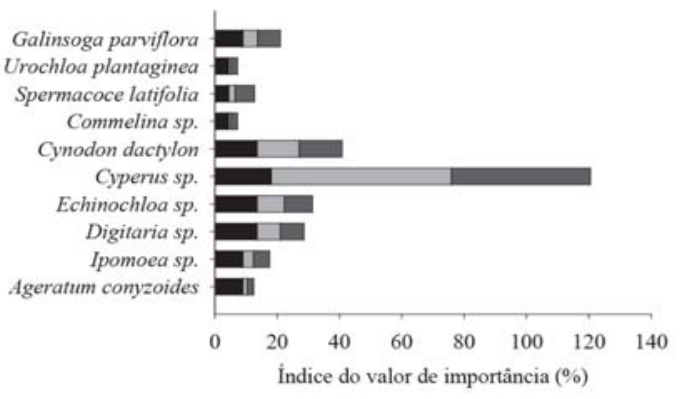

Figura 4 - Índice de valor de importância das plantas daninhas emergidas em resposta aos tratamentos experimentais (presença e ausência da solarização; com e sem capim-elefante) avaliado aos 40 dias após o final da solarização.

Tabela 2 - Massa seca da parte aérea das plantas daninhas (g) aos 10 e 40 dias após o final da solarização (DAS) em função dos tratamentos experimentais

\begin{tabular}{lcc}
\hline & 10 DAS & 40 DAS \\
\hline Solarização & & \\
Ausência & $0,31 \mathrm{a}^{*}$ & $19,76 \mathrm{~b}$ \\
Presença & $0,22 \mathrm{~b}$ & $35,39 \mathrm{a}$ \\
Capim-elefante & & \\
Sem & $0,47 \mathrm{a}$ & $39,00 \mathrm{a}$ \\
Com & $0,06 \mathrm{~b}$ & $16,15 \mathrm{~b}$ \\
CV $(\%)$ & 26,1 & 19,6 \\
\hline
\end{tabular}

* Médias seguidas pela mesma letra nas colunas não diferem entre si pelo teste de Tukey $(\mathrm{p}=0,05)$.

da diversidade de plantas daninhas como constatado no levantamento fitossociológico (Figuras 3 e 4).

A supressão das plantas daninhas decorrente da utilização do capim-elefante pode estar relacionada ao efeito físico proporcionado pela cobertura, interferindo, por exemplo, na germinação de sementes fotoblásticas positivas (Monquero et al., 2009). No estudo fitossociológico, observa-se que a cobertura vegetal evitou a presença do papuã, picão-branco, hortelãdas-roças, picão-roxo, erva de bicho, erva-quente e guanxuma (Figuras 3 e 4), pressupondo que tais espécies pertençam a essa categoria de fotoblastismo com exigência de luz para germinação.

Embora não tenha sido avaliado o tempo de decomposição da palha da cobertura vegetal, possivelmente a utilização do capim triturado acelerou a liberação de compostos orgânicos com potencial alelopático no ambiente, hipotetizando que tais produtos tenham interferido na germinação e desenvolvimento das espécies daninhas. Estudos demonstram o efeito alelopático de compostos fenólicos presentes no capimelefante, reduzindo a germinação de Hedyotis verticillata e Leptochloa chinensis (Norhafizah et al., 2012); e Eleusine indica (Ismail et al., 2015).

\section{CONCLUSÕES}

A adição do capim-elefante na superfície do solo atua como supressor da diversidade de plantas daninhas, como Ageratum conyzoides, Urochloa plantaginea, 
Galinsoga parviflora, Polygonum persicaria, Spermacoce latifolia, Sida sp. e Stachys arvensis.

A solarização aumenta o valor de importância do Cyperus sp. nas parcelas sem a utilização da cobertura vegetal.

A solarização realizada por 20 dias não foi eficiente para o controle das plantas daninhas.

\section{LITERATURA CITADA}

ARORA, A.; YADURAJU, N.T. High-temperature effects on germination and viability of weed seeds in soil. Journal of Agronomy and Crop Science, v.181, n.1, p.35-43, 1998.

CANDIDO, V.; D’ADDABBO, T.; MICCOLIS, V. et al. Effect of different solarizing materials on weed suppression and lettuce response.

Phytoparasitica, v.40, n.2, p.185-194, 2012.

CANDIDO, V.; D’ADDABBO, T.; MICCOLIS, V. et al. Weed control and yield response of soil solarization with different plastic films in lettuce. Scientia Horticulturae, v.130, n.3, p.491-497, 2011.

CORREIA, N.M.; DURIGAN, J.C. Emergência de plantas daninhas em solo coberto com palha de cana-de-açúcar. Planta Daninha, v.22, n.1, p.11-17, 2004.

EL-KEBLAWY, A.; AL-HAMADI, F. Assessment of the differential response of weeds to soil solarization by two methods. Weed Biology and Management, v.9, n.1, p.72-78, 2009.

ELMORE, C.L. Weed control by solarization. In: KATAN, J.; DEVAY, J.E. (Eds). Soil solarization. Boca Raton: CRS, 1991. p.61-72.

EMBRAPA. Centro Nacional de Pesquisa de Solos. Sistema Brasileiro de Classificação de Solos. Brasília: Embrapa Produção de Informação; Rio de Janeiro: Embrapa Solos. 1999. 412p.

FERREIRA, I.C.P.V.; ARAUJO, A.V.; NASCIMENTO, A.L. et al. Cobertura morta e adubação orgânica na produção de alface e supressão de plantas daninhas. Revista Ceres, v.60, n.4, p.582-588, 2013.
HOROWITZ, M.; ROGER, Y.; HERLINGER, G. Solarization for weed control. Weed Science, v.31, n.1, p.170-179, 1983.

ISMAEL, B.S.; TAN, P.W.; CHUAH, T.S. Assessment of the potential allelopathic effects of Pennisetum purpureum Schumach. on the germination and growth of Eleusine indica (L.) Gaertn. Sains Malaysiana, v.44, n.2, p.269-274, 2015.

KANAAN, H.; MEDINA, S.; KRASSNOVSKY, A. et al. Survival of Macrophomina phaseolina s.l. and Verticillium dahliae during solarization as affected by composts of various maturities. Crop

Protection, v.76, n.1, p.108-113, 2015.

KISSMANN, K.G.; GROTH, D. Plantas infestantes e nocivas. 2.ed. São Paulo: BASF. 1997.825p.

KLEIN, E.; KATAN, J.; GAMLIEL, A. Soil suppressiveness to Meloidogyne javanica as induced by organic amendments and solarization in greenhouse crops. Crop Protection, v.39, n.1, p.26-32, 2012.

LAMEGO, F.P.; CARATTI, F.C.; REINEHR, M. et al. Potencial de supressão de plantas daninhas por plantas de cobertura de verão. Comunicata Scientiae, v.6, n.1, p.97-105, 2015.

MARENCO, R.A.; LUSTOSA, D.C. Soil solarization for weed control in carrot. Pesquisa

Agropecuária Brasileira, v.35, n.10, p.20252032, 2000.

MONQUERO, P.A.; AMARAL, L.R.; INÁCIO, E.M. et al. Efeito de adubos verdes na supressão de espécies de plantas daninhas. Planta Daninha, v.27, n.1, p.85-95, 2009.

MÜELLER-DOMBOIS, D.; ELLENBERG, H. Aims and methods of vegetation ecology. New York: John Wiley. 1974. 547p.

NORHAFIZAH, M.Z.; ISMAIL, B.S.; CHUAH, T.S. Herbicidal activity of $P$. purpureum (Napier grass). African Journal of Biotechnology, v.11, n.23, p.6269-6273, 2012.

OLIVEIRA JR., R.S.DE.; CONSTANTIN, J.; INOUE, M.H. Biologia e manejo de plantas daninhas. Curitiba, PR: Omnipax, 2011. 348p. 
PATRÍCIO, F.R.A.; KIMATI, H.; NETO, J.T. et al. Solarização do solo em casa-de-vegetação e campo para o controle de Rhizoctonia solani AG4. Summa Phytopathologica, v.33, n.3, p.245-251, 2007.

RICCI, M.S.F.; ALMEIDA, D.L.; FERNANDES, M.C.A. et al. Efeitos da solarização do solo na densidade populacional da tiririca e na produtividade de hortaliças sob manejo orgânico. Pesquisa Agropecuária Brasileira, v.35, n.11, p.2175-2179, 2000.

ROCHA, G.A.; CARNEIRO, L.C. Solarização do solo associada à incorporação de material orgânico na redução da viabilidade de escleródios. Revista de Ciências Agroambientais, v.14, n.1, p.10-17, 2016.

SETYOWATI, N.; NURJANAH, U.;

SUDJATMIKO, S. et al. Soil solarization with colored plastic mulched influenced weed growth and soil temperature in tropical highland.

International Journal of Agricultural Technology, v.13, n.7,2, p.2053-2063, 2017.
TALEBI, M.R.; GOLPARVAR, A.R. Survey effect of solarization duration and thickness of polyethylene plastic sheets on the characteristics and seed bank of weeds. Scientia

Agriculturae, v.2, n.2, p.26-32, 2013.

THULLEN, R.J.; KEEKEY, P.E. Seed production and germination in Cyperus esculentus and $C$. rotundus. Weed Science, v.27, n.5, p.502-505, 1979.

TREZZI, M.M.; VIDAL, R.A. Potencial de utilização de cobertura vegetal de sorgo e milheto na supressão de plantas daninhas em condição de campo: II - efeitos da cobertura morta. Planta Daninha, v.22, n.1, p.1-10, 2004.

WONG, L.C.; AMBRÓSIO, M.M.Q.; SOUZA, N.L.DE. Sobrevivência de Fusarium oxysporum f. sp. lycopersici Raça 2 submetido a técnica da solarização associada à incorporação de folhas de mandioca. Summa Phytopathologica, v.37, n.2, p.129-133, 2011.

Recebido para publicação em 1/6/2018 e aprovado em 10/3/2019. 